\title{
ANÁLISE DA ESTRUTURA DE UMA COMUNIDADE LENHOSA EM ÁREA DE CERRADO SENSU STRICTO NO MUNICÍPIO DE SENADOR MODESTINO GONÇALVES, NORTE DE MINAS GERAIS, BRASIL ${ }^{1}$
}

\author{
Andreza Viana Neri², João Augusto Alves Meira Neto ${ }^{3}$, Alexandre Francisco da Silva ${ }^{3}$, Sebastião \\ Venâncio Martins ${ }^{4}$ e Márcio Luiz Batista ${ }^{2}$
}

\begin{abstract}
RESUMO - Com a finalidade de conhecer a estrutura de uma comunidade arbórea de uma área de Cerrado, fez-se um estudo fitossociológico no Município de Senador Modestino Gonçalves. Para tal foram delimitadas 30 parcelas de 10 x 20m para levantamento dos dados, utilizando-se como critério de inclusão os indivíduos com circunferência do tronco à altura do solo $(\mathrm{CAS})=10 \mathrm{~cm}$. Foram encontradas 91 espécies de 38 famílias. As espécies que se destacaram como as mais importantes foram Qualea grandiflora, Eriotheca pubescens, Caryocar brasiliense, Byrsonima coccolobaefolia, Myrsine guianensis, Qualea parviflora, Dalbergia miscolobium, Stryphnodendron adstringens, Plathymenia reticulata e Lafoensia pacari. Essas 10 espécies representaram 49,32\% do VI e 51,26\% dos indivíduos amostrados. A área não apresentou espécie com dominância marcante, como mostrou o valor de equabilidade $\left(\mathrm{J}^{\prime}=0,80\right)$. Além de se destacar pela riqueza, o cerrado estudado destacouse também pelos altos valores de densidade $(6.476,67 \mathrm{ind} / \mathrm{ha})$, de área basal $(28,93 \mathrm{~m} / \mathrm{ha})$ e pelo alto índice de diversidade $\left(\mathrm{H}^{\prime}=3,61\right)$.
\end{abstract}

Palavras-chave: Fitossociologia, Cerrado e Vale do Jequitinhonha.

\section{A WOODY COMMUNITY STRUCTURE IN A CERRADO SENSU STRICTO AREA OF THE MUNICIPALITY OF SENADOR MODESTINO GONCALVES, NORTH OF MINAS GERAIS STATE, BRAZIL}

\begin{abstract}
The aim of this work was to study the phytossociological structure of a tree community in a cerrado fragment located in Senador Modestino Gonçalves, MG. A total of $3010 \times 20 \mathrm{~m}$ stands of tree individuals with stem circumferences at the soil level $=10 \mathrm{~cm}$ were sampled, being found 91 species belonging to 38 families. The most important species were Qualea grandiflora, Eriotheca pubescens, Caryocar brasiliense, Byrsonima coccolobaefolia, Myrsine guianensis, Qualea parviflora, Dalbergia miscolobium, Stryphnodendron adstringens, Plathymenia reticulata and Lafoensia pacari. These species represented $49.32 \%$ of the importance value and $51.26 \%$ of the individuals. Besides standing out for its richness, the studied cerrado fragment also outstood out for its high tree density, biomass and diversity.
\end{abstract}

Keywords: Phytosociology, Cerrado and Jequitinhonha Valley.

\footnotetext{
${ }^{1}$ Recebido em 09.03.2006 e aceito para publicação em 20.08.2006.

${ }^{2}$ Programa de Pós-Graduação em Biologia Vegetal da Universidade Federal de Viçosa. E-mail: <andreza_neri@ hotmail.com>.

${ }_{3}^{3}$ Departamento de Biologia Vegetal da Universidade Federal de Viçosa. E-mail: <j.meira@ufv.br>.

${ }^{4}$ Departamento de Engenharia Florestal da Universidade Federal de Viçosa. E-mail: <venancio@ufv.br>.
} 


\section{INTRODUÇÃO}

O bioma Cerrado originalmente cobria cerca de dois milhões de $\mathrm{km}^{2}$ do país, sendo o segundo maior bioma do Brasil (RATTER et al., 1997; MACHADO et al., 2004). Sua extensão compreende uma área core localizada no Planalto Central brasileiro e áreas adjacentes (RIZZINI, 1992). O Cerrado estende-se das margens da Floresta Amazônica até o sul dos Estados de São Paulo e Paraná, ocupando mais de $20^{\circ}$ de latitude e atingindo altitudes de $1.800 \mathrm{~m}$. Na América do Sul, vegetações savânicas ocorrem também na Bolívia, no Paraguai e na Venezuela, porém com baixa riqueza (RIZZINI, 1992; RATTER et al., 1997).

Segundo Coutinho (1978), o Cerrado é caracterizado por cinco fitofisionomias, denominadas campo limpo, campo sujo, campo cerrado, cerrado sensu stricto e cerradão. A ocorrência das diferentes fitofisionomias de Cerrado é freqüentemente dependente das propriedades do solo, profundidade, fertilidade e capacidade de drenagem tanto quanto de interferências humanas (PIVELLO e COUTINHO, 1996). O solo, na maioria das vezes, é distrófico com pH ácido e baixa concentração de cálcio e magnésio disponíveis e alta concentração de alumínio trocável (LOPES e COX, 1977). São solos comumente bem drenados, e onde não ocorre vegetação lenhosa em áreas com alagamento (PENNINGTON et al., 2000).

A variação pedológica no bioma Cerrado proporciona variação estrutural e florística (DURIGAN et al., 2003). Porém, essas diferenças podem ser influenciadas por outros fatores importantes, como o fogo e o clima. As savanas, em geral, possuem certa tolerância ao fogo, devido a adaptações como súber espesso, xilopódio e outras. As freqüentes queimadas que ocorrem no Cerrado causam danos principalmente às espécies lenhosas, mas favorecem as espécies herbáceas (RATTER et al., 1997).

Muitos autores postularam que o fogo seja um dos principais fatores na gênese das diferentes fitofisionomias ou manutenção das áreas mais abertas de Cerrado quando o solo não é fator discriminante (COUTINHO, 1990; PIVELOeCOUTINHO, 1996; RATTER et al., 1997; DEZZEO et al., 2004). Porém, Durigan et al. (2003), numa análise de padrões fitogeográficos do Cerrado Paulista consideraram o clima como principal responsável pelas relações florísticas encontradas, vindo, em seguida, a fertilidade do solo. Segundo esses últimos autores, os padrões regionais encontrados apresentam-se associados à distribuição geográfica das bacias hidrográficas, o que estaria relacionado com a dispersão das espécies.

O que se sabe, contudo, é que o bioma Cerrado é considerado a savana florísticamente mais rica do mundo, em função do seu alto endemismo, sendo uma das áreas brasileiras prioritárias para conservação, juntamente com a Mata Atlântica (MYERS et al., 2000). Assim, deve-se ter atenção não apenas com os cerrados centrais, mas com todo o bioma, inclusive com os cerrados marginais, que podem revelar relações importantes com vegetações vizinhas.

O conhecimento de como as fitofisionomias e as populações de plantas se distribuem no Cerrado tem implicações diretas nas ações de conservação desse bioma. O desmatamento do Cerrado, causado pela expansão da fronteira agropecuária, torna esse momento crucial para que o conhecimento e entendimento das relações ambientais e da distribuição das populações de plantas de Cerrado possam se materializar em ações para a conservação de espécies.

Dessa forma este trabalho procurou conhecer a florística e a estrutura de uma comunidade lenhosa de Cerrado no Município de Senador Modestino Gonçalves, MG, e, assim, contribuir para o conhecimento dessa vegetação numa região onde poucos estudos foram desenvolvidos.

\section{MATERIAL E MÉTODOS}

O Município de Senador Modestino Gonçalves localiza-se na bacia do rio Jequitinhonha (MG), nas coordenadas $43^{\circ} 10^{\prime} \mathrm{W}$ a $43^{\circ} 20^{\prime} \mathrm{W}$ e $17^{\circ} 40^{\prime} \mathrm{S}$ a $17^{\circ} 43^{\prime} \mathrm{S}$, cerca de $370 \mathrm{~km}$ ao norte de Belo Horizonte, MG. O trabalho foi realizado em uma área de reserva legal, localizada na fazenda Ticó, propriedade da Companhia Agrícola Florestal-Santa Bárbara (CAF). A região é caracterizada por relevo plano elevado, com altitude variando de $887 \mathrm{~m}$ a $949 \mathrm{~m}$. O solo da área é do tipo Latossolo Vermelho-Amarelo (EMBRAPA, 1999), a precipitação pluvial média anual é de $1059 \mathrm{~mm}$ e a temperatura do ar média anual de é $21^{\circ} \mathrm{C}$ (CAF, 2001).

A análise de estrutura foi realizada a partir do método de parcelas (MUELLER-DOMBOIS eELLENBERG, 1974). Foram alocadas 30 parcelas de 10 × 20 m, distribuídas ao longo de três linhas paralelas, com distância entre elas de $30 \mathrm{~m}$. As parcelas pertencentes à mesma linha 
foram distanciadas $20 \mathrm{~m}$ entre si. O fato de as árvores do Cerrado apresentarem troncos tortuosos e com bifurcações logo acima do solo impossibilita, muitas vezes, a medida a 1,30 m; em razão dessa característica, muitos autores optam pela medida da circunferência do tronco a $30 \mathrm{~cm}$ ou no nível do solo (FONSECA e SILVAJÚNIOR, 2004; RESENDEetal., 2004; ASSUNÇÃO e FELFILI, 2005; MARIMON JÚNIOR e HARIDASAN, 2005; NERI et al., 2005). Assim, esses autores preferiram medir a circunferência das árvores na altura do solo (CAS), mas tendo sempre o cuidado de medir acima do xilopódio, quando este se apresentou descoberto. Como critério de inclusão foram amostrados os indivíduos com circunferência do caule igual ou superior a $10 \mathrm{~cm}$.

As coletas foram realizadas entre agosto de 2001 e abril de 2002. Todo o material botânico em fenofase fértil foi coletado e, após a identificação, depositado no Herbário do Departamento de Biologia Vegetal da Universidade Federal de Viçosa (VIC), em Viçosa, MG.

Para a florística foi utilizado o sistema de Cronquist (1981), exceto Fabaceae, para a qual se empregou o sistema de Judd et al. (1999). Para confirmação da grafia das espécies, utilizaram-se as informações contidas no site do Missouri Botanical Garden - MOBOT (http:/ /www.mobot.org/W3T/search_vast.html).

$\mathrm{Na}$ análise de estrutura foram avaliados os parâmetros usuais da fitossociologia: densidade, freqüência e dominância absolutas e relativas e valor de importância para as espécies (MUELLER-DOMBOIS e ELLENBERG, 1974), sendo este último a soma dos valores relativos citados anteriormente e representando a importância ecológica de determinada espécie na área de estudo. Esses parâmetros foram estimados pelo programa Fitopac 1 (SHEPHERD, 1994). Também foram calculados o índice de diversidade de Shannon (H') e a equabilidade (J') (PIELOU, 1975).

Para análise de suficiência amostral foram determinados os intervalos de confiança de $90 \%$ de probabilidade (FREESE, 1962; PHILLIP, 1994; FELFILI e SILVA JÚNIOR, 2001), utilizando-se as fórmulas:

a) Média por parcela: $\bar{y}=\frac{\sum_{i=1}^{n} y i}{n}$

b) Variância por parcela: $\mathrm{S}^{2}=\left(\sum_{i=1}^{n} y_{i}^{2}-\left(\sum_{i=1}^{n} y i\right)^{2}\right) / \mathrm{n}-1$ c) Desvio-padrão: $\mathrm{S}=\sqrt{\frac{S^{2}}{n}}$

d) Erro padrão da estimativa: $\mathrm{S}_{-}=\sqrt{\frac{S^{2}}{n}} \cdot f$ $S_{-}$

e) Erro padrão porcentual: $\mathrm{S} \bar{y} \%=\left(\frac{y}{\bar{y}}\right) .100$

f) Fator de correção para populações finitas: $f=1-\frac{n}{N}$ em que:

$\mathrm{Y}=$ área basal por parcela;

$y_{\text {= média de área basal por parcela; }}$

$\mathrm{n}=$ número de parcelas da amostra; e

$\mathrm{N}=$ número total de parcelas que cabem no fragmento.

O esforço amostral foi igualmente avaliado em gráficos, por meio da curva espécie/área, que relaciona o acúmulo de espécies em relação ao aumento da área amostral (PIELOU, 1975). Para a construção do gráfico, as parcelas foram acrescentadas de forma aleatória.

\section{RESULTADOS E DISCUSSÃO}

$\mathrm{Na}$ análise de suficiência amostral foi encontrado um erro-padrão porcentual de $6,15 \%$, para área basal na amostra. Pode-se inferir, então, que a amostragem de 0,60 ha foi suficiente para representar o fragmente estudado, visto que erro-padrão porcentual de até $10 \%$ é interpretado como indicador de suficiência (FELFILI e SILVA JÚNIOR, 2001).

Pela curva espécie/área, pode-se observar que houve tendência à estabilização a partir da $18^{\mathrm{a}}$ parcela, havendo acréscimo mínimo de espécies nas parcelas seguintes. Até a $18^{\mathrm{a}}$ parcela, ou seja, com $60 \%$ de área amostrada, foi possível encontrar $91 \%$ das espécies. A partir desse ponto, houve pequeno incremento no número de espécies a partir da $20^{\mathrm{a}}$ parcela (Figura 1). Apesar de não atingir a assíntota, a curva espécie/ área evidenciou que a área amostral foi suficiente para representar o fragmento.

Foram encontrados 3.886 indivíduos, distribuídos em 91 espécies, 65 gêneros e 38 famílias (Tabela 1). Os indivíduos amostrados representavam densidade estimada de $6.476,67 \mathrm{ind} / \mathrm{ha}$ e área basal de $28,93 \mathrm{~m}^{2} /$ ha $\left(17,36 \mathrm{~m}^{2}\right)$. 


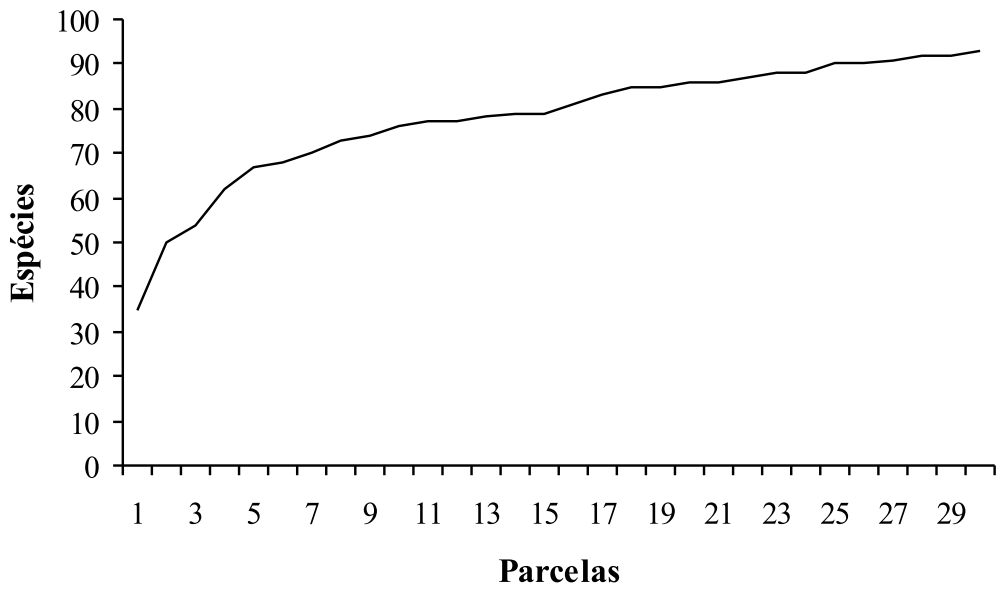

Figura 1 - Curva espécie/área de uma amostra de cerrado sensu stricto do Município de Senador Modestino Gonçalves, MG, Brasil. Figure 1 - Species/area curve of cerrado sensu stricto in Senador Modestino Gonçalves, Minas Gerais, Brazil.

Tabela 1 - Estimativas dos parâmetros fitossociológicos das famílias amostradas nos 0,60 ha de cerrado sensu stricto do Município de Senador Modestino Gonçalves, MG, Brasil, em ordem decrescente do valor de importância (VI\%). NI-número de indivíduos, DA-densidade absoluta, DR-densidade relativa \%, FA-freqüência absoluta, FR-freqüência relativa \%, DoA-dominância absoluta e DoR-dominância relativa \%

Table 1 - Phytosociological parameters estimates of plant families sampled in 0.60 of cerrado sensu stricto, Senador Modestino Gonçalves, Minas Gerais, Brazil, in decreasing order of the importance value (VI\%). NI-Number of individuals, $D A$-absolute density, DR-relative density \%, FA-absolute frequency, FR-relative frequency \%, DoA-absolute dominance and DoR-relative dominance (\%)

\begin{tabular}{|c|c|c|c|c|c|c|c|c|}
\hline FAMÍLIA & N.I. & DA & DR & DoA & DoR & FA & FR & $\% \mathrm{VI}$ \\
\hline Vochysiaceae & 562 & 936,7 & 14,46 & 7,58 & 26,20 & 100,00 & 4,93 & 15,20 \\
\hline Fabaceae & 684 & 1140,0 & 17,60 & 4,40 & 15,21 & 100,00 & 4,93 & 12,58 \\
\hline Bombacaceae & 360 & 600,0 & 9,26 & 2,98 & 10,30 & 96,67 & 4,77 & 8,11 \\
\hline Malpighiaceae & 354 & 590,0 & 9,11 & 1,83 & 6,34 & 100,00 & 4,93 & 6,80 \\
\hline Caryocaraceae & 50 & 83,3 & 1,29 & 3,24 & 11,19 & 73,33 & 3,62 & 5,36 \\
\hline Myrsinaceae & 270 & 450,0 & 6,95 & 1,06 & 3,68 & 90,00 & 4,44 & 5,02 \\
\hline Sapindaceae & 235 & 391,7 & 6,05 & 0,53 & 1,84 & 73,33 & 3,62 & 3,84 \\
\hline Lythraceae & 102 & 170,0 & 2,62 & 0,85 & 2,93 & 86,67 & 4,28 & 3,28 \\
\hline Melastomataceae & 120 & 200,0 & 3,09 & 0,48 & 1,68 & 80,00 & 3,95 & 2,90 \\
\hline Arecaceae & 103 & 171,7 & 2,65 & 0,86 & 2,98 & 56,67 & 2,80 & 2,81 \\
\hline Bignoniaceae & 88 & 146,7 & 2,26 & 0,45 & 1,57 & 90,00 & 4,44 & 2,76 \\
\hline Nyctaginaceae & 92 & 153,3 & 2,37 & 0,51 & 1,75 & 83,33 & 4,11 & 2,74 \\
\hline Myrtaceae & 78 & 130,0 & 2,01 & 0,56 & 1,92 & 83,33 & 4,11 & 2,68 \\
\hline Rubiaceae & 81 & 135,0 & 2,08 & 0,27 & 0,92 & 73,33 & 3,62 & 2,21 \\
\hline Sapotaceae & 55 & 91,7 & 1,42 & 0,80 & 2,76 & 46,67 & 2,30 & 2,16 \\
\hline Apocynaceae & 79 & 131,7 & 2,03 & 0,22 & 0,75 & 73,33 & 3,62 & 2,13 \\
\hline Araliaceae & 63 & 105,0 & 1,62 & 0,15 & 0,53 & 76,67 & 3,78 & 1,98 \\
\hline Ebenaceae & 50 & 83,3 & 1,29 & 0,22 & 0,76 & 73,33 & 3,62 & 1,89 \\
\hline Dilleniaceae & 71 & 118,3 & 1,83 & 0,21 & 0,73 & 53,33 & 2,63 & 1,73 \\
\hline Proteaceae & 69 & 115,0 & 1,78 & 0,36 & 1,26 & 43,33 & 2,14 & 1,72 \\
\hline Annonaceae & 59 & 98,3 & 1,52 & 0,14 & 0,48 & 56,67 & 2,80 & 1,60 \\
\hline
\end{tabular}

R. Árvore, Viçosa-MG, v.31, n.1, p.123-134, 2007 


\begin{tabular}{|c|c|c|c|c|c|c|c|c|}
\hline FAMÍLIA & N.I. & DA & DR & DoA & DoR & FA & FR & $\% \mathrm{VI}$ \\
\hline Meliaceae & 54 & 90,0 & 1,39 & 0,19 & 0,67 & 46,67 & 2,30 & 1,45 \\
\hline Erythroxylaceae & 34 & 56,7 & 0,87 & 0,12 & 0,42 & 56,67 & 2,80 & 1,36 \\
\hline Moracea & 24 & 40,0 & 0,62 & 0,04 & 0,16 & 46,67 & 2,30 & 1,03 \\
\hline Loganiaceae & 11 & 18,3 & 0,28 & 0,28 & 0,98 & 33,33 & 1,64 & 0,97 \\
\hline Guttifera & 19 & 31,7 & 0,49 & 0,11 & 0,38 & 40,00 & 1,97 & 0,95 \\
\hline Celastraceae & 24 & 40,0 & 0,62 & 0,13 & 0,46 & 26,67 & 1,32 & 0,80 \\
\hline Connaraceae & 14 & 23,3 & 0,36 & 0,03 & 0,12 & 36,67 & 1,81 & 0,76 \\
\hline Asteracea & 27 & 45,0 & 0,69 & 0,07 & 0,26 & 23,33 & 1,15 & 0,70 \\
\hline Euphorbiaceae & 11 & 18,3 & 0,28 & 0,05 & 0,18 & 30,00 & 1,48 & 0,65 \\
\hline Opiliaceae & 11 & 18,3 & 0,28 & 0,04 & 0,14 & 23,33 & 1,15 & 0,52 \\
\hline Lauraceae & 19 & 31,7 & 0,49 & 0,09 & 0,32 & 10,00 & 0,49 & 0,43 \\
\hline Styracaceae & 4 & 6,7 & 0,10 & 0,01 & 0,04 & 13,33 & 0,66 & 0,27 \\
\hline Indeterminadas & 3 & 5,0 & 0,08 & 0,01 & 0,02 & 10,00 & 0,49 & 0,20 \\
\hline Burseraceae & 2 & 3,3 & 0,05 & 0,00 & 0,01 & 6,67 & 0,33 & 0,13 \\
\hline Verbenaceae & 1 & 1,7 & 0,03 & 0,01 & 0,02 & 3,33 & 0,16 & 0,07 \\
\hline Rutaceae & 1 & 1,7 & 0,03 & 0,00 & 0,02 & 3,33 & 0,16 & 0,07 \\
\hline Flacourtiaceae & 1 & 1,7 & 0,03 & 0,00 & 0,01 & 3,33 & 0,16 & 0,07 \\
\hline Anacardiaceae & 1 & 1,7 & 0,03 & 0,00 & 0,01 & 3,33 & 0,16 & 0,07 \\
\hline
\end{tabular}

O trecho de Cerrado estudado apresentou alto valor de densidade quando comparado com outros trabalhos realizados nesse bioma (RIBEIRO et al., 1985; ROSSI et al., 1998; SANTOS, 2000; COSTAeARAÚJO, 2001; SILVA et al., 2002; ANDRADE et al., 2002). Segundo Felfili et al. (1994), as amplitudes de densidade de Cerrado sensu stricto na região de Goiás e do Distrito Federal (DF) estão entre 664-1396 ind/ha. Dessa forma, o valor encontrado aqui está mais próximo daqueles citados pelos referidos autores para Cerradão (960-2.082 ind/ha). Contudo, em razão desses valores o Cerrado estudado pode ser considerado um Cerrado sensu stricto denso e não um Cerradão, pois, apesar do alto valor de densidade, não apresenta características fisionômicas florestais; ao contrário, a área apresenta uma flora herbáceo-subarbustiva bem representativa. $\mathrm{O}$ fato de a área ter sido protegida do fogo e não ter sido alvo de corte seletivo certamente contribuiu para a alta densidade encontrada. Outro fator que muito favoreceu a alta densidade foi a medida da circunferência na altura do solo.

A área basal de $28,93 \mathrm{~m}^{2} /$ ha também foi mais alta que as encontradas por outros autores (ANDRADE et al., 2002; RIBEIRO et al., 1985). Costa e Araújo (2001) encontraram valores inferiores (2001) na Reserva de Panga, Uberlândia, MG, tanto para Cerrado sensu stricto (9,63 $\left.\mathrm{m}^{2} / \mathrm{ha}\right)$ quanto para o Cerradão (17,06 m²/ha). Valor de área basal próximo ao do presente estudo foi encontrado por Santos (não publicado) no Parque Estadual do Lajeado, Palmas, TO (23,62 m²/ha). Nesse caso, o autor utilizou o mesmo critério de inclusão, ao contrário de Costa e Araújo (2001), que usaram perímetro superior ou igual a $15 \mathrm{~cm}$ medidos à altura de 1,30 m do solo. O fato de a área apresentar valor de área basal superior aos obtidos nos trabalhos supracitados não quer dizer que ele não represente uma área de Cerrado sensu stricto, pois essa diferença se deve, principalmente, ao critério de inclusão e ao local onde foram medidos os caules que proporcionaram maior densidade e, como consequiência, alta área basal.

Alguns estudos têm mostrado que a diversidade em Cerrado sensu stricto varia de 3,16 a 3,73 (FELFILI et al., 1993, 1997; SANTOS, 2000; COSTA e ARAÚJO 2001; FELFILI e SILVA JÚNIOR, 2001; MEIRA NETO e SAPORETTI Jr, 2002; ASSUNÇÃO e FELFILI, 2004). Em Senador Modestino Goçalves, a diversidade avaliada pelo índice de Shannon ( $\mathrm{H}^{\prime}$ ) foi de 3,61 e a equabilidade (J), 0,80. Comparando com os índices de diversidade encontrados em outras áreas de Cerrado, verificouse que esse estava entre os mais altos. A equabilidade (J) de 0,80 encontrada indicou que os indivíduos estão bem distribuídos entre as espécies, exceto por Qualea grandiflora, Eriotheca pubescens, Myrsine guianensis e Byrsonima coccolobaefolia, que se mostraram abundantes na área. Aliado ao índice de diversidade,

R. Árvore, Viçosa-MG, v.31, n.1, p.123-134, 2007 
verificou-se também alta riqueza de espécies em relação às outras áreas de mesma fisionomia (FELFILI e SILVA JÚNIOR, 2001;ASSUNÇÃOe FELFILI, 2004; FONSECA e SILVA JÚNIOR, 2004; MARIMON JUNIOR e HARIDASAN, 2005).

Neste estudo, as 10 famílias mais importantes perfizeram $73,01 \%$ dos indivíduos amostrados. Fabaceae apresentou-se com o maior número de indivíduos, seguida por Vochysiaceae, Bombacaceae, Malpighiaceae, Myrsinaceae, Sapindaceae, Melastomataceae, Lythraceae e Caryocaraceae (Tabela 1).

Fabaceae destacou-se, principalmente, em razão de sua alta densidade, em decorrência do alto número de indivíduos amostrados das subfamílias Mimosoideae e Papilionoideae, sendo a subfamília Caesalpinioideae pouco representada. Fabaceae é uma família que se destaca não só em áreas de Cerrado, mas também em outras formações vegetais, tanto em riqueza específica quanto em valores de importância. Heringer et al. (1977) consideraram Fabaceae uma das famílias predominantes na flora savanícola, já que está presente com altas densidades em todas as fitofisionomias de Cerrado. Segundo Cordeiro (2000), Fabaceae, pela capacidade de nodulação de suas espécies, apresenta maior adaptabilidade em regiões com baixo teor de nitrogênio, sendo essa uma vantagem sobre as demais espécies, principalmente no Cerrado, onde o solo, na maioria das vezes, é pobre em nutrientes.

Vochysiaceae igualmente se destacou com relação à densidade, e esses altos valores foram em decorrência do grande número de indivíduos de Qualea grandiflora, que representou $11,30 \%$ dos indivíduos amostrados (Tabela 2). As outras espécies dessa família, Q. parviflora, $Q$. multiflora e $Q$. cordata, tiveram menor contribuição. Essa família se destacou com a maior densidade, em outros estudos, como em Cerrado sensu stricto e Cerradão (COSTA e ARAÚJO, 2001), e em Cerrado denso (ANDRADE et al., 2002). Essa família, juntamente com Fabaceae e Malpighiaceae, foi encontrada em todas as unidades amostrais, apresentando, assim, os maiores valores em freqüência.

Tabela 2 - Parâmetros fitossociológicos das espécies amostradas nos 0,60 ha de cerrado sensu stricto do Município de Senador Modestino Gonçalves, MG, Brasil, em ordem decrescente do valor de importância (VI\%). NI-número de indivíduos, DA-densidade absoluta, DR-densidade relativa \%, DoA dominância absoluta, DoR-dominância relativa \%, FA-frequiência absoluta e FR-freqüência relativa \%

Table 2 - Phytosociological parameters of plant species of cerrado sensu stricto, 0.60 hectare, Senador Modestino Gonçalves, Minas Gerais, Brazil, in decreasing order of importance value (VI\%). NI-Number of individuals, DA-absolute density, $D R$-relative density \%, FA-absolute frequency, FR-relative frequency \%, DoA-absolute dominance and DoR-relative dominance $(\%)$

\begin{tabular}{|c|c|c|c|c|c|c|c|c|c|}
\hline ESPECIE & $\mathrm{NI}$ & $\mathrm{NU}$ & DA & DR & DoA & DoR & FA & FR & $\mathrm{VI} \%$ \\
\hline Qualea grandiflora Mart. & 439 & 30 & 731,7 & 11,30 & 4,9383 & 17,07 & 100,00 & 3,23 & 10,53 \\
\hline Eriotheca pubescens (Mart. \& Zucc.) Schott \& Endl. & 360 & 29 & 600,0 & 9,26 & 2,9789 & 10,30 & 96,67 & 3,12 & 7,56 \\
\hline Caryocar brasiliense Cambess. & 50 & 22 & 83,3 & 1,29 & 3,2366 & 11,19 & 73,33 & 2,37 & 4,95 \\
\hline Byrsonima coccolobifolia Kunth & 230 & 30 & 383,3 & 5,92 & 1,2787 & 4,42 & 100,00 & 3,23 & 4,52 \\
\hline Myrsine guianensis (Aubl.) Kuntze & 270 & 27 & 450,0 & 6,95 & 1,0651 & 3,68 & 90,00 & 2,90 & 4,51 \\
\hline Qualea parviflora Mart. & 102 & 22 & 170,0 & 2,62 & 2,3878 & 8,25 & 73,33 & 2,37 & 4,41 \\
\hline Dalbergia miscolobium Benth. & 173 & 28 & 288,3 & 4,45 & 0,7808 & 2,70 & 93,33 & 3,01 & 3,39 \\
\hline Stryphnodendron adstringens (Mart.) Coville & 144 & 27 & 240,0 & 3,71 & 0,9921 & 3,43 & 90,00 & 2,90 & 3,35 \\
\hline Plathymenia reticulata Benth. & 122 & 25 & 203,3 & 3,14 & 1,1962 & 4,13 & 83,33 & 2,69 & 3,32 \\
\hline Lafoensia pacari A. St.-Hil. & 102 & 26 & 170,0 & 2,62 & 0,8483 & 2,93 & 86,67 & 2,80 & 2,78 \\
\hline Bowdichia virgilioides Kunth & 126 & 19 & 210,0 & 3,24 & 0,7483 & 2,59 & 63,33 & 2,04 & 2,62 \\
\hline Syagrus flexuosa (Mart.) Becc. & 103 & 17 & 171,7 & 2,65 & 0,8629 & 2,98 & 56,67 & 1,83 & 2,49 \\
\hline Matayba sp & 169 & 16 & 281,7 & 4,35 & 0,3611 & 1,25 & 53,33 & 1,72 & 2,44 \\
\hline Guapira noxia (Netto) Lundell & 65 & 25 & 108,3 & 1,67 & 0,4459 & 1,54 & 83,33 & 2,69 & 1,97 \\
\hline Miconia ligustroide (DC.) Naudin & 61 & 21 & 101,7 & 1,57 & 0,2589 & 0,90 & 70,00 & 2,26 & 1,57 \\
\hline Aspidosperma tomentosum Mart. & 69 & 22 & 115,0 & 1,78 & 0,1670 & 0,58 & 73,33 & 2,37 & 1,57 \\
\hline Miconia albicans (Sw.) Triana & 59 & 22 & 98,3 & 1,52 & 0,2260 & 0,78 & 73,33 & 2,37 & 1,56 \\
\hline Didymopanax macrocarpus (Cham. \& Schltdl.) Seem. & 62 & 23 & 103,3 & 1,60 & 0,1533 & 0,53 & 76,67 & 2,47 & 1,53 \\
\hline Acosmium dasycarpum (Vogel) Yakovlev & 43 & 22 & 71,7 & 1,11 & 0,3000 & 1,04 & 73,33 & 2,37 & 1,50 \\
\hline
\end{tabular}

R. Árvore, Viçosa-MG, v.31, n.1, p.123-134, 2007 
Quadro 2-Cont.

Table 2 - Cont.

\begin{tabular}{|c|c|c|c|c|c|c|c|c|c|}
\hline$\overline{\text { ESPECIE }}$ & $\mathrm{NI}$ & $\mathrm{NU}$ & DA & DR & DoA & DoR & FA & FR & VI\% \\
\hline Roupala montana Aubl. & 69 & 13 & 115,0 & 1,78 & 0,3632 & 1,26 & 43,33 & 1,40 & 1,48 \\
\hline Davilla rugosa Poir. & 71 & 16 & 118,3 & 1,83 & 0,2115 & 0,73 & 53,33 & 1,72 & 1,43 \\
\hline Tabebuia ochracea (Cham.) Standl. & 45 & 20 & 75,0 & 1,16 & 0,2798 & 0,97 & 66,67 & 2,15 & 1,43 \\
\hline Diospyros hispida A. DC. & 49 & 21 & 81,7 & 1,26 & 0,2172 & 0,75 & 70,00 & 2,26 & 1,42 \\
\hline Tocoyena formosa (Cham. \& Schltdl.) K. Schum. & 50 & 20 & 83,3 & 1,29 & 0,1724 & 0,60 & 66,67 & 2,15 & 1,34 \\
\hline Zeyheria digitalis (Vell.) L. B. Smith \& Sandwith & 42 & 21 & 70,0 & 1,08 & 0,1722 & 0,60 & 70,00 & 2,26 & 1,31 \\
\hline Pouteria ramiflora (Mart.) Radlk. & 42 & 12 & 70,0 & 1,08 & 0,4433 & 1,53 & 40,00 & 1,29 & 1,30 \\
\hline Byrsonima verbascifolia (L.) DC. & 36 & 17 & 60,0 & 0,93 & 0,3242 & 1,12 & 56,67 & 1,83 & 1,29 \\
\hline Duguetia furfuracea (A. St.-Hil.) Saff. & 57 & 17 & 95,0 & 1,47 & 0,1346 & 0,47 & 56,67 & 1,83 & 1,25 \\
\hline Psidium pohlianum $\mathrm{O}$. Berg & 35 & 17 & 58,3 & 0,90 & 0,2621 & 0,91 & 56,67 & 1,83 & 1,21 \\
\hline Cabralea canjerana (Vell.) Mart. & 54 & 14 & 90,0 & 1,39 & 0,1940 & 0,67 & 46,67 & 1,51 & 1,19 \\
\hline Magonia pubescens A. St.-Hil. & 45 & 11 & 75,0 & 1,16 & 0,1116 & 0,39 & 36,67 & 1,18 & 0,91 \\
\hline Heteropterys byrsonimifolia A. Juss. & 47 & 8 & 78,3 & 1,21 & 0,1293 & 0,45 & 26,67 & 0,86 & 0,84 \\
\hline Enterolobium gummiferum (Mart.) J.F. Macbr. & 23 & 11 & 38,3 & 0,59 & 0,1959 & 0,68 & 36,67 & 1,18 & 0,82 \\
\hline Erythroxylum daphnites Mart. & 27 & 13 & 45,0 & 0,69 & 0,1011 & 0,35 & 43,33 & 1,40 & 0,81 \\
\hline Strychnos pseudoquina A. St.-Hil. & 11 & 10 & 18,3 & 0,28 & 0,2824 & 0,98 & 33,33 & 1,08 & 0,78 \\
\hline Brosimum gaudichaudii Trécul & 24 & 14 & 40,0 & 0,62 & 0,0457 & 0,16 & 46,67 & 1,51 & 0,76 \\
\hline Pouteria torta (Mart.) Radlk. & 13 & 6 & 21,7 & 0,33 & 0,3543 & 1,22 & 20,00 & 0,65 & 0,73 \\
\hline Guapira ferruginea (Klotzsch ex Choisy) Lundell & 22 & 13 & 36,7 & 0,57 & 0,0496 & 0,17 & 43,33 & 1,40 & 0,71 \\
\hline Byrsonima intermedia A. Juss. & 21 & 12 & 35,0 & 0,54 & 0,0447 & 0,15 & 40,00 & 1,29 & 0,66 \\
\hline Austroplenckia populnea (Reissek) Lundell & 24 & 8 & 40,0 & 0,62 & 0,1317 & 0,46 & 26,67 & 0,86 & 0,64 \\
\hline Eugenia dysenterica DC. & 19 & 10 & 31,7 & 0,49 & 0,1028 & 0,36 & 33,33 & 1,08 & 0,64 \\
\hline Randia armata (Sw.) DC. & 26 & 8 & 43,3 & 0,67 & 0,0793 & 0,27 & 26,67 & 0,86 & 0,60 \\
\hline Hymenaea stigonocarpa Mart. ex Hayne & 19 & 9 & 31,7 & 0,49 & 0,0932 & 0,32 & 30,00 & 0,97 & 0,59 \\
\hline Qualea multiflora Mart. & 18 & 7 & 30,0 & 0,46 & 0,1412 & 0,49 & 23,33 & 0,75 & 0,57 \\
\hline Rourea induta Planch. & 14 & 11 & 23,3 & 0,36 & 0,0349 & 0,12 & 36,67 & 1,18 & 0,55 \\
\hline Kielmeyera coriacea Mart. \& Zucc. & 13 & 9 & 21,7 & 0,33 & 0,0715 & 0,25 & 30,00 & 0,97 & 0,52 \\
\hline Mimosa verrucosa Benth. & 18 & 8 & 30,0 & 0,46 & 0,0630 & 0,22 & 26,67 & 0,86 & 0,51 \\
\hline Pera glabrata (Schott) Poepp. ex Baill. & 11 & 9 & 18,3 & 0,28 & 0,0529 & 0,18 & 30,00 & 0,97 & 0,48 \\
\hline Eugenia sp1 & 8 & 6 & 13,3 & 0,21 & 0,1529 & 0,53 & 20,00 & 0,65 & 0,46 \\
\hline Agonandra brasiliensis Miers ex Benth. \& Hook. f. & 11 & 7 & 18,3 & 0,28 & 0,0399 & 0,14 & 23,33 & 0,75 & 0,39 \\
\hline Ocotea felix Coe-Teixeira & 19 & 3 & 31,7 & 0,49 & 0,0922 & 0,32 & 10,00 & 0,32 & 0,38 \\
\hline Lychnophora salicifolia Mart. & 15 & 4 & 25,0 & 0,39 & 0,0436 & 0,15 & 13,33 & 0,43 & 0,32 \\
\hline Eugenia aurata $\mathrm{O}$. Berg & 9 & 6 & 15,0 & 0,23 & 0,0174 & 0,06 & 20,00 & 0,65 & 0,31 \\
\hline Machaerium opacum Vogel & 7 & 6 & 11,7 & 0,18 & 0,0139 & 0,05 & 20,00 & 0,65 & 0,29 \\
\hline Aspidosperma macrocarpon Mart. & 9 & 4 & 15,0 & 0,23 & 0,0488 & 0,17 & 13,33 & 0,43 & 0,28 \\
\hline Kielmeyera petiolares Mart. & 6 & 5 & 10,0 & 0,15 & 0,0377 & 0,13 & 16,67 & 0,54 & 0,27 \\
\hline Sapindaceae 1 & 13 & 3 & 21,7 & 0,33 & 0,0439 & 0,15 & 10,00 & 0,32 & 0,27 \\
\hline Cupania vernalis Cambess. & 8 & 5 & 13,3 & 0,21 & 0,0162 & 0,06 & 16,67 & 0,54 & 0,27 \\
\hline Vernonia sp & 11 & 3 & 18,3 & 0,28 & 0,0293 & 0,10 & 10,00 & 0,32 & 0,24 \\
\hline Nea theifera Orsted & 5 & 5 & 8,3 & 0,13 & 0,0106 & 0,04 & 16,67 & 0,54 & 0,23 \\
\hline Qualea cordata (Mart.) Spreng. & 3 & 2 & 5,0 & 0,08 & 0,1114 & 0,39 & 6,67 & 0,22 & 0,23 \\
\hline Myrcia multiflora (Lam.) DC. & 5 & 4 & 8,3 & 0,13 & 0,0147 & 0,05 & 13,33 & 0,43 & 0,20 \\
\hline Styrax camporum Pohl & 4 & 4 & 6,7 & 0,10 & 0,0119 & 0,04 & 13,33 & 0,43 & 0,19 \\
\hline Banisteriopsis malifolia (Nees \& Mart.) B. Gates & 5 & 3 & 8,3 & 0,13 & 0,0178 & 0,06 & 10,00 & 0,32 & 0,17 \\
\hline Erythroxylum tortuosum Mart. & 5 & 3 & 8,3 & 0,13 & 0,0132 & 0,05 & 10,00 & 0,32 & 0,17 \\
\hline Alibertia sessilis (Vell.) K. Schum. & 4 & 3 & 6,7 & 0,10 & 0,0141 & 0,05 & 10,00 & 0,32 & 0,16 \\
\hline Heteropterys eglandulosa A. Juss. & 6 & 2 & 10,0 & 0,15 & 0,0173 & 0,06 & 6,67 & 0,22 & 0,14 \\
\hline Byrsonima sp & 3 & 3 & 5,0 & 0,08 & 0,0061 & 0,02 & 10,00 & 0,32 & 0,14 \\
\hline Indeterminadas & 3 & 3 & 5,0 & 0,08 & 0,0060 & 0,02 & 10,00 & 0,32 & 0,14 \\
\hline Sclerolobium paniculatum Vogel & 5 & 2 & 8,3 & 0,13 & 0,0099 & 0,03 & 6,67 & 0,22 & 0,13 \\
\hline
\end{tabular}


Quadro 2- Cont.

Table 2-Cont.

\begin{tabular}{|c|c|c|c|c|c|c|c|c|c|}
\hline ESPECIE & NI & $\mathrm{NU}$ & DA & DR & DoA & DoR & FA & FR & VI\% \\
\hline Banisteriopsis stellaris (Griseb.) B. Gates & 3 & 2 & 5,0 & 0,08 & 0,0120 & 0,04 & 6,67 & 0,22 & 0,11 \\
\hline Erythroxylum gonoclados (Mart.) O.E. Schulz & 2 & 2 & 3,3 & 0,05 & 0,0074 & 0,03 & 6,67 & 0,22 & 0,10 \\
\hline Annona dioica A. St.-Hil. & 2 & 2 & 3,3 & 0,05 & 0,0047 & 0,02 & 6,67 & 0,22 & 0,09 \\
\hline Protium heptaphyllum (Aubl.) Marchand & 2 & 2 & 3,3 & 0,05 & 0,0028 & 0,01 & 6,67 & 0,22 & 0,09 \\
\hline Bauhinia rufa (Bong.) Steud. & 2 & 1 & 3,3 & 0,05 & 0,0032 & 0,01 & 3,33 & 0,11 & 0,06 \\
\hline Lantana sp & 1 & 1 & 1,7 & 0,03 & 0,0070 & 0,02 & 3,33 & 0,11 & 0,05 \\
\hline Zanthoxylum rhoifolium Lam. & 1 & 1 & 1,7 & 0,03 & 0,0056 & 0,02 & 3,33 & 0,11 & 0,05 \\
\hline Myrcia sp & 1 & 1 & 1,7 & 0,03 & 0,0049 & 0,02 & 3,33 & 0,11 & 0,05 \\
\hline Diospyros sericea A. DC. & 1 & 1 & 1,7 & 0,03 & 0,0041 & 0,01 & 3,33 & 0,11 & 0,05 \\
\hline Senna rugosa (G. Don) H.S. Irwin \& Barneby & 1 & 1 & 1,7 & 0,03 & 0,0032 & 0,01 & 3,33 & 0,11 & 0,05 \\
\hline Cybistax antisyphilitica (Mart.) Mart. & 1 & 1 & 1,7 & 0,03 & 0,0031 & 0,01 & 3,33 & 0,11 & 0,05 \\
\hline Gochnatia pulchra Cabrera & 1 & 1 & 1,7 & 0,03 & 0,0024 & 0,01 & 3,33 & 0,11 & 0,05 \\
\hline Himatanthus obovatus (Müll. Arg.) Woodson & 1 & 1 & 1,7 & 0,03 & 0,0021 & 0,01 & 3,33 & 0,11 & 0,05 \\
\hline Byrsonima salzmanniana A. Juss. & 1 & 1 & 1,7 & 0,03 & 0,0020 & 0,01 & 3,33 & 0,11 & 0,05 \\
\hline Dalbergia brasiliensis Vogel & 1 & 1 & 1,7 & 0,03 & 0,0019 & 0,01 & 3,33 & 0,11 & 0,05 \\
\hline Casearia sylvestris Sw. & 1 & 1 & 1,7 & 0,03 & 0,0019 & 0,01 & 3,33 & 0,11 & 0,05 \\
\hline Tapirira guianensis Aubl. & 1 & 1 & 1,7 & 0,03 & 0,0016 & 0,01 & 3,33 & 0,11 & 0,05 \\
\hline Myrtaceae 1 & 1 & 1 & 1,7 & 0,03 & 0,0015 & 0,01 & 3,33 & 0,11 & 0,05 \\
\hline Banisteriopsis campestris (A. Juss.) Little & 1 & 1 & 1,7 & 0,03 & 0,0014 & 0,00 & 3,33 & 0,11 & 0,05 \\
\hline Didymopanax morototoni (Aubl.) Decne. \& Planch. & 1 & 1 & 1,7 & 0,03 & 0,0013 & 0,00 & 3,33 & 0,11 & 0,05 \\
\hline Palicourea rigida Kunth & 1 & 1 & 1,7 & 0,03 & 0,0013 & 0,00 & 3,33 & 0,11 & 0,05 \\
\hline Banisteriopsis anisandra (A. Juss.) B. Gates & 1 & 1 & 1,7 & 0,03 & 0,0013 & 0,00 & 3,33 & 0,11 & 0,05 \\
\hline
\end{tabular}

O maior valor de dominância relativa (DoR) foi obtido, também, por Vochysiaceae seguida de Fabaceae. Em razão do alto valor de DoR, que foi bem superior ao de Fabaceae, o táxon obteve o maior valor de importância (VI).

Vochysiaceae tem sido considerada a família mais representativa e está sempre entre as mais importantes quando se trata de áreas de Cerrado. Essa família se destaca não apenas quanto aos parâmetros fitossociológicos (RIBEIRO et al., 1985; FELFILI et al., 1993; ARAÚJO et al., 1997; SANTOS - Dados não publicados; COSTAe ARAÚJO, 2001; SILVAet al., 2002; BALDUÍNO et al., 2005), mas também em relação à fitomassa lenhosa aérea. Vochysiaceae foi considerada a família mais importante do Cerrado sensu stricto por Ribeiro e Haridasan (1984), devido ao fato de as suas espécies serem acumuladoras de alumínio, conferindolhes vantagens competitivas nos solos ácidos desse bioma (FELFILI et al., 1993; HARIDASAN, 2000).

Outra família significativa do Cerrado é Caryocaraceae, que sempre está entre as mais importantes desse bioma, sendo bem representada na maioria dos estudos. Neste trabalho, esse táxon se destacou pelo alto valor de dominância relativa de Caryocar brasiliense.
Apesar dos poucos indivíduos amostrados, essa família apresentou alta dominância absoluta e relativa (Tabela1); os dados aqui contemplados são semelhantes àqueles discutidos por Ratter et al. (1996, 2003), onde Caryocaraceae está presente em mais de $60 \%$ das áreas analisadas de Cerrado, mostrando o predomínio da distribuição da família no bioma.

Espécies que se destacam com altos valores de importância (VI) são espécies que possuem maior sucesso na exploração dos recursos oferecidos pelo ambiente (ANDRADE et al., 2002). No estudo em questão, as espécies que apresentaram os maiores valores de importância foram: Qualea grandiflora, Eriotheca pubescens, Caryocar brasiliense, Byrsonima coccolobaefolia, Myrsine guianensis, Qualea parviflora, Dalbergia miscolobium, Stryphnodendron adstringens, Plathymenia reticulata e Lafoensia pacari (Figura 2). Essas 10 espécies representaram 49,32\% do VI e 51,26\% dos indivíduos amostrados (Tabela 2). Adominância exercida por algumas poucas espécies em florestas tropicais é comum, e isso também se estende às áreas de Cerrado, como pode ser observado em vários estudos (RIBEIRO et al., 1985; GUARIMNETOetal., 1994; MARIMONetal., 1998; SILVA e SOARES, 2002; SILVA et al., 2002; ANDRADE et al., 2002; BALDUINO et al., 2005). 


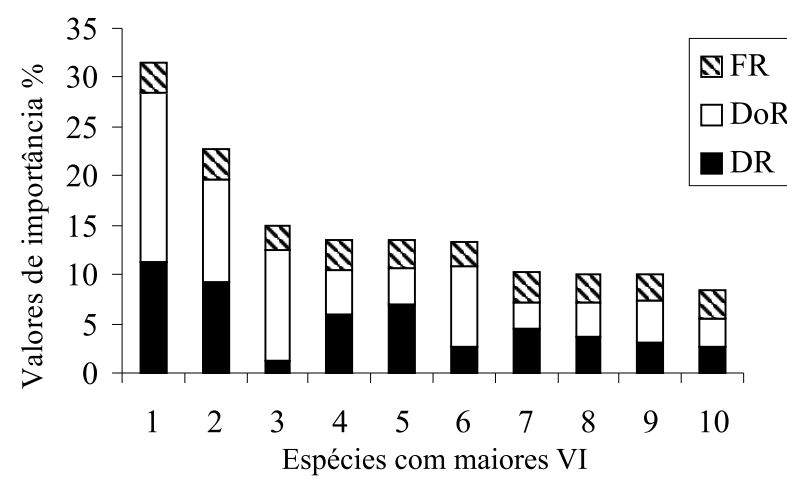

Figura 2 - Espécies com maiores valores de importância (VI\%) numa área de cerrado sensu stricto no município de Senador Modestino Gonçalves, MG, Brasil. 1 (Qualea grandiflora), 2 (Eriotheca pubescens), 3 (Caryocar brasiliense), 4 (Byrsonima coccolobaefolia), 5 (Myrsine guianensis), 6 (Qualea parviflora), 7 (Dalbergia miscolobium), 8 (Stryphnodendron adstringens), 9 (Plathymenia reticulata) e 10 (Lafoensia pacari).

Figure 2-Species with larger importance values (VI\%) in the area of cerrado sensu stricto, Senador Modestino Gonçalves, Minas Gerais, Brazil. 1 (Qualea grandiflora), 2 (Eriotheca pubescens), 3 (Caryocar brasiliense), 4 (Byrsonima coccolobaefolia), 5 (Myrsine guianensis), 6 (Qualea parviflora), 7 (Dalbergia miscolobium), 8 (Stryphnodendron adstringens), 9 (Plathymenia reticulata) e 10 (Lafoensia pacari).

A maioria das espécies que obtiveram os maiores valores de importância se caracteriza pela sua ampla distribuição no Cerrado e estão entre as mais importantes do bioma.

Das principais espécies que se destacaram em valor de importância, Qualea grandiflora, Q. parviflora, Lafoensia pacari, Byrsonima coccolobaefolia, Caryocar brasiliense e Plathymenia reticulata ocorreram em mais de $50 \%$ das 376 áreas de Cerrado avaliadas por Ratter et al. (2003).

Qualea grandiflora destacou-se em todos os outros parâmetros fitossociológicos avaliados. $\mathrm{O}$ alto valor de dominância conferida a essa espécie se deve não somente ao grande número de indivíduos encontrado, mas à área basal total (Tabela 2). Ratter et al. (1996, 2003) destacaram $Q$. grandiflora como uma espécie que, além de acentuada dominância, possui ampla distribuição no Cerrado e está presente em $85 \%$ dos trabalhos avaliados por esses autores. Nas áreas amostradas, essa espécie foi encontrada tanto em áreas de Cerrado sensu stricto quanto em áreas de Cerradão, assim como em áreas de campo cerrado.

Eriotheca pubescens, com o segundo maior valor de importância, apresentou grande número de indivíduos, o que lhe conferiu o segundo maior valor de densidade relativa e altos valores de dominância e freqüência relativas (Tabela 2). Ao contrário das demais espécies que obtiveram alto VI, E. pubescens não ocorre com grande freqüência no Cerrado (RATTER et al., 2003) e, quando ocorre, geralmente não está entre as 10 mais importantes da comunidade (ROSSI et al., 1998; COSTA e ARAÚJO, 2001; ANDRADE et al., 2002), apesar de ser uma espécie peculiar desse bioma (RIZZINI, 1963; HERINGER et al., 1977).

Caryocar brasiliense, que apresentou menor densidade relativa entre as 10 espécies mais importantes, obteve o terceiro lugar em VI, em razão, principalmente, de sua dominância relativa (Tabela 2). Essa espécie possui ampla distribuição e vem sendo considerada uma das principais do Cerrado (GOODLAND, 1979), destacandose também em outros trabalhos (RIBEIRO et al., 1985; RATTER et al., 1996, 2003; ARAÚJO et al., 1997; FELFILI e SILVA JÚNIOR, 2001; SILVA et al., 2002).

Byrsonima coccolobaefolia, que também possui ampla distribuição no Cerrado (RATTER et al., 1996, 2003), ficou em quarto lugar em valor de importância. Essa espécie apresentou alta frequiência, estando presente em todas as unidades amostrais. Ela também exibiu altos valores de densidade e dominância relativas. $\mathrm{O}$ alto valor de dominância de $B$. coccolobaefolia deveuse, especialmente, ao seu grande número de indivíduos (Tabela 2).

Myrsine guianensis, a quinta espécie mais importante, apresentou alta densidade relativa. Esse parâmetro, juntamente com a freqüência relativa, foi o principal responsável pelo alto VI. O alto valor de dominância dessa espécie também foi conseqüência do elevado valor de densidade relativa, pois os indivíduos amostrados possuíam pequena circunferência (Tabela 2).

Qualea parviflora é quase tão ocorrente em áreas de Cerrado sensu stricto e Cerradão quanto $Q$. grandiflora. Neste trabalho, a espécie se destacou principalmente no parâmetro de dominância relativa e obteve o sexto maior valor de importância (Tabela 2), estando bem distribuída na área e presente em mais de $70 \%$ das parcelas.

R. Árvore, Viçosa-MG, v.31, n.1, p.123-134, 2007 
Outras espécies que se destacaram em valor de importância (VI) foram Dalbergia miscolobium, Stryphnodendron adstringens, Plathymenia reticulata e Lafoensia pacari (Tabela 2).

Apesar de ser um Cerrado marginal, a área estudada se assemelhava muito floristicamente às áreas core, sobretudo nas regiões estudadas em Minas Gerais, destacando-se ainda pela alta riqueza florística, e isso reforça a necessidade de se conhecer melhor a região norte do Cerrado em Minas Gerais.

\section{AGRADECIMENTOS}

À Fundação de Amaro à Pesquisa do Estado de Minas Gerais (FAPEMIG) e à Companhia Agrícola Florestal-Santa Bárbara (CAF-Santa Bárbara), pelo apoio financeiro, e aos funcionários da CAF-Santa Bárbara de Carbonita, MG pelo auxílio nos trabalhos de campo.

\section{REFERÊNCIAS}

ASSUNÇÃO, S.L.; FELFILI, J.M. Fitossociologia de um fragmento de cerrado sensu stricto na APA de Paranoá, DF, Brasil. Acta Botanica Brasilica, v.18, n.4, p.903-910, 2004.

ANDRADE, L. A. Z.; FELFILI, J. M.; VIOLATTI, L. Fitossociologia de uma área de cerrado denso na RECOR-IBGE, Brasília-DF. Acta Botanica Brasílica, v.16, n.2, p.225-240, 2002.

ARAÚJO, G. M. et al. Estrutura comunitária de vinte áreas de cerrados residuais no município de Uberlândia, MG. Daphne, v.7, n.2, p.7-14, 1997.

BALDUÍNO, A P. C. et al. Fitossociologia e análise comparativa de composição florística do Cerrado na Floana de Paraopeba-MG. Revista Árvore, v.29, n.1, p.25-34, 2005.

\section{COMPANHIA AGRÍCOLA FLORESTAL SANTA} BÁRBARA LTDA - CAF. Região de

Carbonita. Belo Horizonte: Companhia Agrícola Floresta Santa Bárbara, 2001.

CORDEIRO, L. Fixação de nitrogênio em leguminosas ocorrentes no cerrado. In: KLEIN, A. L. (Org.). Eugen Warming e o Cerrado brasilleiro: um século depois. São Paulo: Universidade Estadual de São Paulo, 2000. p.131-145.

R. Árvore, Viçosa-MG, v.31, n.1, p.123-134, 2007
COSTA, A. A.; ARAúJO, G. M. Comparação da vegetação arbórea de cerradão e de cerrado na Reserva do Panga, Uberlândia, Minas Gerais. Acta Botanica Brasilica, v.15, n.1, p.63-72, 2001.

COUTINHO, L.M. O conceito de cerrado. Revista Brasileira de Botânica, v.1, p.17-23, 1978.

COUTINHO,L. M. Fire in the ecology of the Brazilian cerrado. In: GOLDAMMER, J. G. (Ed.). Fire in the Tropical Biota: Ecosystem processes and global challenges. Berlin: Springer-Velag, 1990. p.82-105.

CRONQUIST, A. An integrated system of classification of flowering plants. New York: Columbia University, 1981. 1262p.

DEZZEO, N. et al. Changes in soil properties and vegetation characteristics along a forestsavanna gradient in southern Venezuela.

Forest Ecology and Management, v.200, p.183-193, 2004.

DURIGAN, G. et al. Padrões fitogeográficos do cerrado paulista sob uma perspectiva regional. Hoehnea, v.30, n.1, p.39-51, 2003.

EMPRESA BRASILEIRA DE PESQUISA AGROPECUÁRIA - EMBRAPA. Sistema brasileiro de classificação de solos. Brasília: 1999.

FELFILI, J. M. et al. Análise comparativa da florística e fitossociologia do vegetação arbórea do cerrado sensu stricto na Chapada Pratinha, DF-Brasil. Acta Botanica Brasílica, v.6, n.2, p.27-46, 1993.

FEFILI, J. M. et al. Projeto biogeografia do bioma Cerrado: vegetação e solos. Caderno de Geociências, v.12, n.4, p.75-166, 1994.

FELFILI, J. M. et al. Comparação do cerrado (stricto sensu) nas Chapadas Pratinhae dos Veadeiros. In: LEITE, L. L., SAITO, T. H. (Ed.). CONGRESSO DE ECOLOGIA DO BRASIL, 3., 1996, Brasília. Anais... Brasília: Universidade de Brasília, 1997. p.6-11. 
FELFILI, J. M.; SILVA JÚNIOR, M.C.

Biogeografia do Bioma Cerrado: estudo fitofisionômico na Chapada do Espigão Mestre do São Francisco. Brasília: Universidade de Brasília, 2001. 152p.

FREESE, F. Elementary forest sampling. Washington: USDA, 1962.91p.

FONSECA, M.S.; SILVA JÚNIOR, M.C. Fitossociologia e similaridade florística entre trechos de Cerrado sentido restrito em interflúvio e em vale no Jardim Botânico de Brasília, DF. Acta Botanica Brasilica, v.18, n.1, p.19-30, 2004.

GUARIM NETO, G.; GUARIM, V. L. M. S.; PRANCE, G. T. Struture and floristic composition of the trees of na área of cerrado near Cuibá, Mato Grosso. Kew Bulletin, v.49, n.30, p.499-509, 1994.

GOODLAND, R. Análise ecológica da vegetação do cerrado. In GOODLAND, R.; FERRI, M. G. Ecologia do Cerrado. Belo Horizonte: Itatiaia, 1979. p.61-171.

HARIDASAN, M. Nutrição mineral das plantas nativas do Cerrado - grupos funcionais. In: CAVALCANTI, T. B.; WALTER, B. M. T. (Org.). Tópicos atuais em botânica: palestras convidadas. CONGRESSO NACIONAL DE BOTÂNICA, 51. 2000, Brasília. Anais... Brasília: Embrapa Recursos Genéticos e Biotecnologia/Sociedade Botânica do Brasil, 2000. p.159-164.

HERINGER, E. P. et al. A flora do Cerrado. In: FERRI, M. G. (Ed.). SIMPÓSIO SOBRE O CERRADO, 4., 1977, São Paulo. Anais... São Paulo: Universidade de São Paulo, 1977. p.303-316.

http://mobot.mobot.org/W3T/search_vast.html.

JUDD, W. S. et al. Plant systematics: a phylogenetic approach. Suderland: Sinner Associates, 1999. 464p.

LOPES, A. S.; COX, F. R. A survey of the fertility status of surface soils under 'cerrado' vegetation in Brazil. Soil Science Society of

America Journal, v.41, p.741-747, 1977.
MACHADO, R.B. et al. Estimativas de perda da área do Cerrado Brasileiro. Brasília: Conservação Internacional. 2004. 26p. (Relatório Técnico não publicado).

MARIMON, B.S.; VALLERA, R.F.; MARIMON JÚNIOR, B.H. Fitossociologia de uma área de cerrado de encosta em Nova Xavantina, Mato Grosso. Boletim do Herbário Ezechias Paulo Heringer, v.3, p.82-101, 1998.

MARIMON JUNIOR, B.H.; HARIDASAN, M. Comparação da vegetação arbórea e características edáficas de um cerradão e um cerrado sensu stricto em áreas adjacentes em solos distróficos no leste de Mato Grosso, Brasil. Acta Botanica Brasílica, v.19, n.4, p.913-926.

MEIRA NETO, J. A. A.; SAPORETTI JÚNIOR, A. W. Composição florística em cerrado no Parque Nacional da Serra do Cipó, MG. Revista Árvore, v.26, n.5, p.645-648, 2002.

MUELLER-DOMBOIS, D.; ELLENBERG, H. Aims and methods of vegetation ecology. New York: John Wiley \& Sons, 1974. 547p.

MYERS, N. et al. Biodiversity hotspots for conservation priorities. Nature, v.403, p.853-858, 2000.

NERI, A.V. et al. regeneração de espécies nativas lenhosas sob plantio de Eucalyptus em área de Cerrado na Floresta Nacional de Paraopeba, MG, Brasil. Acta Botanica Brasilica, v.19, n.2, p.369-376, 2005.

PENNINGTON, R. T.; PRADO, D. E.; PENDRY, C. A. Neotropical seasonally dry forests and quaternary vegetation changes. Journal of Biogeography, v.27, p.261-273, 2000.

PHILLIP, M. S. Measuring trees and forests. Oxford: CAB international, 1994. 310p.

PIELOU, E. C. Ecology diversity. New York: John Wiley \& Sons, 1975. 165p.

PIVELLO, V. R.; COUTINHO, L. M. A qualitative successional model to assist in the management of Brazilian cerrados.

Forest Ecology and Management, v.87, p.127-138, 1996.

R. Árvore, Viçosa-MG, v.31, n.1, p.123-134, 2007 
RATTER, J. A. et al. Analysis of the floristic composition of the brasilian cerrado vegetation II: comparison of the woody vegetation of 98 areas. Edinburgh Journal of Botany, v.53, n.2, p.153-180, 1996.

RATTER, J. A.; RIBEIRO, J. F. \& BRIDGEWATER, $\mathrm{S}$. The Brazilian cerrado vegetation and threats to its biodiversity. Annals of Botany, v.80, p.223-230, 1997.

RATTER, J. A., BRIDGEWATER, S.; RIBEIRO, J. F. Analysis of the floristic compositionof the Brasilian Cerrado vegetation. III: comparison of the woody vagetation of 376 areas. Edinburgh Journal of Botany, v.60, n.1, p.57-109, 2003.

RESENDE, I.L.M. et al. A comunidade vegetal e as características abióticas de um campo de murundu em Uberlândia, MG. Acta Botanica

Brasílica, v. 18, n.1, p.9-18, 2004.

RIBEIRO, J. F.; HARIDASAN, M. Comparação fitossociológica de um cerrado denso e um cerradão em solos distróficos no Distrito Federal. In: CONGRESSO NACIONAL DE BOTÂNICA, 35 ., 1984, Manaus. Anais... Manaus: Sociedade de Botânica do Brasil, 1984. p.342-347.

RIBEIRO, J. F.; SILVA, J. C. S.; BATMANIAN, G. J. Fitossociologia de tipos fisionômicos de cerrados em Planaltina-DF. Revista Brasileira de Botânica, v.8, n.1, p.131-142, 1985.

RIZZINI, C. T. Tratado de fitogeografia do Brasil. São Paulo: Universidade de São Paulo, 1992. 747p.
RIZZINI, C. T. A flora do Cerrado: análise florística das savanas centrais. In: FERRI, M. G. (Ed.), SIMPÓSIO SOBRE O CERRADO, 1963, São Paulo. Anais... São Paulo: Universidade de São Paulo, 1963. p.125-177.

ROSSI, C. V.; SILVA JUNIOR, M. C.; SANTOS, C. E. N. Fitossociologia do estrato arbóreo do Cerrado (Sensu Stricto) no Parque Ecológico Norte, BrasíliaDF. Boletim do Herbário Ezechias Paulo Heringer, v.2, p.49-56, 1998.

SANTOS, E. R. Análise florística e estrutura fitossociológica da vegetação lenhosa de um trecho de cerrado stricto sensu do Parque Estadual do Lajeado, Palmas-TO. 2000. 64f. Dissertação (Mestrado em Biologia Vegetal) - Universidade Federal de Viçosa, Viçosa, MG, 2000.

SHEPHERD, G. J. Fitopac 1. Manual do usuário. Campinas: Universidade Estadual de Campinas, 1994.96p.

SILVA, L. O. et al. Levantamento florístico e fitossociológico em duas áreas de cerrado sensu stricto no Parque Estadual da Serra de Caldas Novas, Goiás. Acta Botanica Brasilica, v.16, n.1, p.43-53, 2002.

SILVA, L. A.; SOARES, J. J. Levantamento fitossociológico em um fragmento de floresta estacional semidecídua, no município de São Carlos, SP. Acta Botanica Brasilica, v.16, n.2, p.205-216, 2002. 